\title{
Missed diagnosis of influenza B virus due to nucleoprotein sequence mutations, Singapore, April 2011
}

H K Lee', C K Lee ${ }^{1}$, T P Loh², D Chiang³ , E S Koay,4, J W Tang (julian_wt_tang@nuhs.edu.sg)1

1. Molecular Diagnosis Centre, Department of Laboratory Medicine, National University Hospital, Singapore

2. Clinical Chemistry Division, Department of Laboratory Medicine, National University Hospital, Singapore

3. Microbiology Division, Department of Laboratory Medicine, National University Hospital, Singapore

4. Department of Pathology, Yong Loo Lin School of Medicine, National University of Singapore, Singapore

Lee HK, Lee CK, Loh TP, Chiang D, Koay ES, Tang JW. Missed diagnosis of influenza B virus due to nucleoprotein sequence mutations, Singapore, April 2011. Euro Surveill. 2011;16(33):pii=19943. Available online: http://www.eurosurveillance.org/ViewArticle.aspx?Articleld=19943

Article published on 18 August 2011

A new influenza B variant was discovered in Singapore in April 2011 during diagnostic testing of a 3-yearold boy with respiratory symptoms. Influenza B virus was isolated from culture and confirmed by standard immunofluorescence testing, but was not detected by the routine, in-house influenza screening reversetranscription polymerase chain reaction assay that targets the nucleoprotein (NP) gene. Subsequent sequencing investigations demonstrated that several other published assays targeting NP could also fail to detect this novel variant.

Influenza A and B viruses are detectable all year round in tropical Singapore, with overlapping peak incidences of these viruses occurring at approximately the same time, i.e. during calendar weeks 12-30 in the years where such peaks are discernible [1]. Surveillance testing for influenza viruses of samples from patients with influenza-like illness (ILI) obtained from both community clinics and those admitted to hospital has been ongoing in our routine diagnostic laboratory (Molecular Diagnosis Centre, National University Hospital, Singapore) since the emergence of the influenza $A\left(\mathrm{H}_{1} \mathrm{~N}_{1}\right) 2009$ pandemic.
On 12 April 2011 (week 18), influenza B was detected by direct immunofluorescence staining (Light Diagnostic Respiratory Viral Screen and Identification DFA Kit, Chemicon International, Millipore, Temecula, CA, USA) and virus culture of a nasopharyngeal swab collected from a 3-year-old boy, who was admitted to our hospital with ILI. The specimen tested negative on our diagnostic influenza A/B duplex real-time, reverse-transcription polymerase chain reaction (rRT-PCR) assay.

This in-house assay was designed to detect all human influenza A and B subtypes and targets the nucleoprotein (NP) gene of influenza B viruses. To investigate the cause of this discrepancy, the NP gene of this influenza $B$ virus isolate was sequenced directly from the clinical sample and compared with that of a patient infected with the routinely detectable, circulating seasonal influenza B virus (B/Singapore/5/2011, GenBank accession number CYo93581) isolated during April 2011. Three missense mutations (G158A, G697A, G1465A) and 16 synonymous single nucleotide substitutions, were noted in this putative Singaporean influenza B variant (B/Singapore/1/2011, GenBank accession number CYo93580). Further sequencing of the haemagglutinin

\section{TABLE 1}

Primer and probe sequences of influenza B assays presenting mismatches to the influenza B/Singapore/1/2011 virus sequence

\begin{tabular}{|c|c|c|c|c|}
\hline Primer/probe name & Sequence ( $5^{\prime}$ to $\left.3^{\prime}\right)$ & $\begin{array}{l}\text { Nucleotide position } \\
\left(5^{\prime} \text { to } 3^{\prime}\right)^{\mathrm{a}}\end{array}$ & $\begin{array}{l}\text { Sequence } \\
\text { orientation }\end{array}$ & Reference \\
\hline \multicolumn{5}{|l|}{ rRT-PCR } \\
\hline FluBNA916F20 & CCAGGGATTGCAGACATTGA & $916-935$ & Forward & This study \\
\hline FluBNA968U24 & TTGTTAGGCCCTCTGTGGCRAGCA & $968-991$ & Forward & This study \\
\hline NPB5'705 & AACTGGTGTTGCGATCAAAGGAGGTGG & $717-743$ & Forward & {$[8,9]$} \\
\hline \multicolumn{5}{|c|}{ RT-PCR (including nested) } \\
\hline FluABC2 & ATKGCGCWYRAYAMWCTYARRTCTTCAWAIGC & $1208-1177$ & Reverse & [11] \\
\hline FluAB3 (Nested) & GATCAAGTGAKMGRRAGYMGRAAYCCAGG & $892-920$ & Forward & [11] \\
\hline FluB4 (Nested) & CTTAATATGGAAACAGGTGTTGCCATATT & $1118-1090$ & Reverse & [11] \\
\hline
\end{tabular}

rRT-PCR: real-time, reverse-transcription PCR.

Nucleotides of the primer or probe mismatching the sequence of the possible new variant influenza B/Singapore/1/2011 are in bold.

${ }^{a}$ Based on influenza B virus (B/Singapore/1/2011) nucleoprotein gene, GenBank accession: CYo93580. 
(HA) and neuraminidase (NA) genes of this virus is planned, to determine if this virus is a possible new variant or whether these changes in its NP gene simply represent the minor antigenic drift phenomenon typical of all influenza viruses.

This NP variation may affect the ability of other PCRbased in-house assays to detect this virus in clinical samples as two of the single nucleotide substitutions (G975A and C978T) were present within the probe-binding region of our in-house assay (Table 1). Therefore we reviewed previously published influenza $B$ assays that target the NP gene. Of the ten influenza B assays identified [2-11], three $[8,9,11]$ had primer and/or probe sequences presenting mismatches that may reduce assay sensitivity. The affected primer/probe sequences are summarised in Table 1.

The probe-mismatch in our in-house assay for this new $B /$ Singapore/1/2011 virus most probably explains the discrepancy with the results from virus isolation and immunofluorescent staining for the case presented, and is a good example of the importance of maintaining virus culture for clinical testing (at least in reference laboratories), despite rapid developments in molecular diagnostic techniques.

To be able to reliably detect the possible new influenza $B$ variant, an additional probe was designed, whilst retaining the previous probe to allow the continued detection of the influenza B/Singapore/5/2011virus that may still be circulating in the Singaporean population. As part of the validation assay for this modified assay, we tested an additional 200 clinical specimens that had been reported as negative for influenza A/B viruses during April 2011, using the previous version of this in-house rRT-PCR screening assay.

Three additional influenza $B$ positive cases were detected that had identical NP sequences to the virus variant isolated from the 3-year-old boy. These three cases included a 75 -year-old patient with metabolic syndrome presenting with community-acquired pneumonia and heart failure, a 60-year-old patient who presented with non-neutropaenic febrile illness, and a routine community surveillance specimen (no clinical history provided). All three hospitalised patients were discharged shortly after admission with no clinical complications.

With the use of the modified assay, of the five influenza $B$ viruses detected during April 2011, four were of the possible new variant. Pairwise comparisons between more recent and older reference (vaccine-related) influenza B NP sequences (Table 2) show that the NP sequence of the possible new influenza $B$ variant virus ( $B /$ Singapore/1/2011) presents highest similarity to the NP sequence of the most recently circulating $B /$ North Carolina/WRAIR1582P/2009 strain (Victoria lineage). Interestingly, however, when compared to the NP sequences of the older $\mathrm{B} /$ Yamagata/16/88 (Yamagata lineage) and of the $B /$ Victoria/2/87 reference strain (Victoria lineage), the possible new influenza $B$ variant virus NP sequence presents higher identity to the $\mathrm{B} /$ Yamagata/16/88 (Table 2). As the NP gene is relatively well evolutionarily conserved compared to the HA and NA genes, only additional sequencing of the HA and NA genes from the possible new influenza $B$ variant virus detected in this study, and additional Singaporean influenza B viruses, will clarify whether a novel influenza B variant, not previously seen in Singapore, is emerging, and whether it is of either the $\mathrm{B} /$ Victoria/2/87 or the $\mathrm{B} /$ Yamagata/16/88 lineage.

The World Health Organization (WHO)'s current seasonal influenza vaccine recommendations for influenza $B$ vaccine composition for the southern hemisphere [12] and for the approaching influenza season in the northern hemisphere [13] are the same. Both include the $B / B$ risbane/60/2008-like virus, which is of the $B /$ Victoria/2/87 lineage. In China, nevertheless, the B/ Yamagata/16/88 lineage viruses have been predominating from September 2010 to January 2011 [13]. China is a popular and frequent destination for many Singaporean Chinese, who have family connections there, so it is likely that influenza $B$ viruses from both the $\mathrm{B} /$ Victoria/2/87 and $\mathrm{B} /$ Yamagata/16/88 lineages are co-circulating in the Singaporean population. Additional sequencing of Singaporean influenza B viruses will allow an assessment of any potential vaccine mismatch between the seasonal influenza vaccine composition and any possible new variants arising in these locally circulating viruses.

\section{TABLE 2}

Degree of similarity between full-length nucleoprotein gene sequences of Singaporean and reference influenza B viruses, based on pairwise comparisons

\begin{tabular}{|l|c|c|c|c|} 
& $\begin{array}{c}\text { B/Yamagata/16/88 } \\
\text { L49385 }\end{array}$ & $\begin{array}{c}\text { B/Victoria/2/87 } \\
\text { AF100359 }\end{array}$ & $\begin{array}{c}\text { B/Florida/4/2006 } \\
\text { (Yamagata lineage) } \\
\text { CY033879 }\end{array}$ & $\begin{array}{c}\text { B/North Carolina/WRAIR1582P/2009 } \\
\text { (Victoria lineage) } \\
\text { CY069566 }\end{array}$ \\
\hline $\begin{array}{l}\text { B/Singapore/1/2011 } \\
\text { CYo93580 }\end{array}$ & 0.9684 & 0.9449 & 0.9851 & 0.9902 \\
\hline $\begin{array}{l}\text { B/Singapore/5/2011 } \\
\text { CYo93581 }\end{array}$ & 0.9730 & 0.9483 & 0.9879 & 0.9925 \\
\hline
\end{tabular}

a GenBank accession number. 


\section{Conclusions}

Our preliminary report highlights two important issues. First, at local level, it is important for diagnostic virology laboratories using molecular techniques to be constantly vigilant about the possibility of emerging novel virus variants that may decrease the sensitivity of their frontline screening assays [14]. This requires the availability of parallel testing using viral isolation by culture. In addition, this report highlights the need for diagnostic laboratories to test any in-house assay that is developed, based on a published method, on their own population of circulating viruses and adapt their primers and probes accordingly, so as to minimise the risk of diagnostic misses.

Second, from a public health perspective, it is essential for diagnostic and research laboratories worldwide to continuously update and share gene sequences of (possible novel) local viruses on public databases. This allows laboratories to develop diagnostic assays for new variants and the in-silico assessment of their sensitivity and specificity, i.e. multiple-sequence alignment of the available uploaded sequences against primer/probe sequences.

These two issues are not unrelated. While the clinical management of an individual patient may be adversely affected by a diagnostic 'miss' of a novel, emerging virus, at a global population level, due to the limited time window for the inclusion of novel influenza virus antigens into the WHO recommended composition of the seasonal influenza virus vaccines, timely dissemination of data on such novel virus variants is critical to try to avoid vaccine mismatches as much as possible. Vaccine mismatches can have a serious impact on seasonal influenza morbidity and mortality, particularly in vulnerable groups that rely on vaccines for protection against annual seasonal influenza epidemics worldwide.

\section{References}

1. Loh TP, Lai FY, Tan ES, Thoon KC, Tee NW, Cutter J, et al. Correlations between clinical illness, respiratory virus infections and climate factors in a tropical paediatric population. Epidemiol Infect. 2011:1-11.

2. Muradrasoli S, Mohamed N, Belak S, Czifra G, Herrmann $B$, Berencsi $G$, et al. Broadly targeted triplex real-time PCR detection of influenza $A, B$ and $C$ viruses based on the nucleoprotein gene and a novel "MegaBeacon" probe strategy. J Virol Methods. 2010;163(2):313-22.

3. Chi XS, Hu A, Bolar TV, Al-Rimawi W, Zhao P, Tam JS, et al. Detection and characterization of new influenza $B$ virus variants in 2002. J Clin Microbiol. 2005;43(5):2345-9.

4. Lam WY, Yeung AC, Tang JW, Ip M, Chan EW, Hui M, et al. Rapid multiplex nested PCR for detection of respiratory viruses. J Clin Microbiol. 2007;45(11):3631-40.

5. Templeton KE, Scheltinga SA, Beersma MF, Kroes AC, Claas EC. Rapid and sensitive method using multiplex real-time PCR for diagnosis of infections by influenza $A$ and influenza $B$ viruses, respiratory syncytial virus, and parainfluenza viruses 1, 2, 3, and 4. J Clin Microbiol. 2004;42(4):1564-9.

6. Gunson RN, Collins TC, Carman WF. Real-time RT-PCR detection of 12 respiratory viral infections in four triplex reactions. I Clin Virol. 2005;33(4):341-4.

7. Yang Y, Gonzalez R, Huang F, Wang W, Li Y, Vernet G, et al. Simultaneous typing and HA/NA subtyping of influenza $A$ and $B$ viruses including the pandemic influenza $A / H_{1} N_{1} 2009$ by multiplex real-time RT-PCR. J Virol Methods. 2010;167(1):37-44.
8. Smith AB, Mock V, Melear R, Colarusso P, Willis DE. Rapid detection of influenza $A$ and $B$ viruses in clinical specimens by Light Cycler real time RT-PCR. J Clin Virol. 2003;28(1):51-8.

9. Lassauniere R, Kresfelder T, Venter M. A novel multiplex real-time RT-PCR assay with FRET hybridization probes for the detection and quantitation of 13 respiratory viruses. J Virol Methods. 2010;165(2):254-60.

10. Letant SE, Ortiz II, Bentley Tammero LF, Birch IM, Derlet RW, Cohen S, et al. Multiplexed reverse transcriptase PCR assay for identification of viral respiratory pathogens at the point of care. J Clin Microbiol. 2007;45(11):3498-505.

11. Coiras MT, Perez-Brena P, Garcia ML, Casas I. Simultaneous detection of influenza $A, B$, and $C$ viruses, respiratory syncytial virus, and adenoviruses in clinical samples by multiplex reverse transcription nested-PCR assay. J Med Virol. 2003;69(1):132-44.

12. World Health Organization (WHO). Recommended viruses for influenza vaccines for use in the 2011 southern hemisphere influenza season. Geneva:WHO. 29 Sep 2010. Available from: http://www.who.int/influenza/vaccines/virus/ recommendations/201009_Recommendation.pdf

13. World Health Organization (WHO). Recommended composition of influenza virus vaccines for use in the 2011-2012 northern hemisphere influenza season. Geneva:WHO. 17 Feb 2011. Available from: http://www.who.int/csr/disease/ influenza/2011_02_recommendation.pdf

14. Trevino C, Bihon S, Pinsky BA. A synonymous change in the influenza A virus neuraminidase gene interferes with PCR-based subtyping and oseltamivir resistance mutation detection. J Clin Microbiol. 2011;49(8):3101-2. 